\title{
The first determination of Generalized Polarizabilities of the proton by a Virtual Compton Scattering experiment
}

J. Roche ${ }^{1 \dagger}$, J.M. Friedrich ${ }^{2}$, D. Lhuillier ${ }^{1}$, P. Bartsch ${ }^{2}$, D. Baumann ${ }^{2}$, J. Berthot ${ }^{4}$, P.Y. Bertin ${ }^{4}$, V. Breton ${ }^{4}$, W.U. Boeglin ${ }^{2 \ddagger}$, R. Böhm² ${ }^{2}$ N. D’Hose ${ }^{1 *}$, S. Derber ${ }^{2}$, N. Degrande ${ }^{3}$, M. Ding ${ }^{2}$, M.O. Distler ${ }^{2}$, J.E. Ducret ${ }^{1}$, I. Ewald ${ }^{2}$, H. Fonvieille ${ }^{4}$, J. Friedrich ${ }^{2}$, P.A.M. Guichon ${ }^{1}$, H. Holvoet ${ }^{3}$, Ch.E. Hyde-Wright ${ }^{5}$, P. Jennewein ${ }^{2}$, M. Kahrau ${ }^{2}$, S. Kerhoas ${ }^{1}$, K.W. Krygier ${ }^{2}$, B. Lannoy ${ }^{3}$, A. Liesenfeld ${ }^{2}$, C. Marchand ${ }^{1}$, D. Marchand ${ }^{1 \S}$, J. Marroncle ${ }^{1}$, J. Martino ${ }^{1}$, H. Merkel ${ }^{2}$, P. Merle ${ }^{2}$, G. De Meyer ${ }^{3}$, J. Mougey ${ }^{\text {*** }}$, U. Müller ${ }^{2}$, R. Neuhausen ${ }^{2}$, Th. Pospischil ${ }^{2}$, G. Quemener ${ }^{4 * *}$, O. Ravel ${ }^{4 \dagger \dagger}$, Y. Roblin ${ }^{4}$, D. Rohe ${ }^{2}$, G. Rosner ${ }^{2 \ddagger \ddagger}$, D. Ryckbosch ${ }^{3}$, H. Schmieden ${ }^{2}$, G. Tamas ${ }^{2}$, M. Tytgat ${ }^{3}$, M. Vanderhaeghen ${ }^{2}$, L. Van Hoorebeke ${ }^{3}$, R. Van de Vyver ${ }^{3}$, J. Van de Wiele ${ }^{6}$, P. Vernin ${ }^{1}$, A. Wagner ${ }^{2}$, Th. Walcher ${ }^{2}$ and M. Weiss ${ }^{2}$

${ }^{1}$ CEA Saclay, DSM/DAPNIA/SPhN, 91191 Gif-sur-Yvette Cedex, France

${ }^{2}$ Institut für Kernphysik, Universität Mainz, 55099 Mainz, Germany

${ }^{3}$ FWO and SSF, University of Gent, Proeftuinstraat 86, 9000 Gent, Belgium

${ }^{4}$ LPC de Clermont-Fd, IN2P3-CNRS, Université Blaise Pascal, 63177 Aubiere Cedex, France

${ }^{5}$ Old Dominion University, Norfolk, VA23529, U.S.A.

${ }^{6}$ IPN d'Orsay, IN2P3-CNRS, Université de Paris Sud, 91406 Orsay Cedex, France

\begin{abstract}
Absolute differential cross sections for the reaction $e p \rightarrow$ $e p \gamma$ have been measured at a four-momentum transfer with virtuality $Q^{2}=0.33 \mathrm{GeV}^{2}$ and polarization $\epsilon=0.62$ in the range 33.6 to $111.5 \mathrm{MeV} / \mathrm{c}$ for the momentum of the outgoing photon in the photon-proton center of mass frame. The experiment has been performed with the high resolution spectrometers at the Mainz Microtron MAMI. From the photon angular distributions, two structure functions which are a linear combination of the generalized polarizabilities have been determined for the first time.
\end{abstract}

PACS numbers: 13.60.Fz, 14.20.Dh, 13.10.+q, 25.30.Rw

Polarizabilities are fundamental quantities that characterize the response of a composite system to static or slowly varying external electric or magnetic fields. For the proton, Compton scattering of real photons has been used to determine the electric and magnetic polarizabilities [1]. In this letter, we report measurements of Compton scattering of virtual photons, leading to the first determination of generalized polarizabilities (GPs). Virtual Compton scattering (VCS) off the proton refers to the reaction $\gamma^{*} p \rightarrow p \gamma$ where $\gamma^{*}$ stands for an incoming virtual photon of four-momentum squared $Q^{2}$. This reaction is experimentally accessed through photon electroproduction $e p \rightarrow e p \gamma$. We study this reaction in the regime where the produced photon has a small enough energy that its electric (E) and magnetic (M) fields look constant over the size of the nucleon. In this regime the reaction can be interpreted as electron scattering on a nucleon placed in a quasi-constant applied EM field [2]. The induced motion of the nucleon as a whole can be eliminated thanks to a low energy theorem [3], so one is left with the deformation, due to the applied field, of the nucleon internal currents $\delta J^{\mu}(r)$ and the electron scattering measures its Fourier transform $\delta J^{\mu}(Q)$. To lowest order in $\alpha_{Q E D}, \delta J^{\mu}(Q)$ is linear in the applied field and the 6 coefficents of proportionality are the GPs [2, 4, 5]. When $Q^{2}=0$ two of them reduce to the usual polarizabil- ities $\alpha_{E}$ and $\beta_{M}$ measured in real Compton scattering [1]. A measurement of how the nucleon's internal current distribution is deformed by an external EM field will yield valuable information about its non perturbative structure. This is illustrated by the quite different results obtained for the GPs according to the used model [6-10]. As an example of new information provided by VCS and which could not be obtained either from ordinary electron scattering or real Compton scattering, consider the case of Coulomb scattering on a nucleon placed in a magnetic field. The action of the latter is mainly to flip the spin of the quarks, which hardly modifies the charge density. Therefore the corresponding GPs (namely $P^{(11,00) 1}$ and $P^{(11,02) 1}$ in Eq. 3) receive no contribution from the quarks. This is confirmed by actual calculations. As a consequence the value of these GPs should be mainly determined by the pion cloud.

First indications of the feasibility of a VCS experiment were reported in [1]. However only recently, with the advent of high-intensity continuous beam electron accelerators, a thorough experimental investigation of the VCS process became possible. This letter reports on the first VCS experiment dedicated to the GPs which has been performed at the Mainz Microtron MAMI.

The general theoretical framework for VCS has been extensively described by Guichon et al. [4,2] and by Drechsel et al. [5] and only the relevant issues will be discussed here. In the reaction $e p \rightarrow e p \gamma$, the final photon can be emitted either by the electron, which is called the Bethe-Heitler (BH) process, or by the proton. The latter process is the VCS process which consists of Born and Non-Born amplitudes; while the Born amplitude depends only on static properties of the proton (charge, mass, form factors), the Non-Born amplitude contains dynamical internal structure information in terms of GPs. Despite of the strong dominance of the $\mathrm{BH}$ process, the VCS process can be measured under appropriately cho- 
sen kinematics through the interference with the dominant $\mathrm{BH}$ amplitude. In the zero-energy limit of the final photon, the cross section is independent of the dynamical nucleon structure [3], and can be evaluated using only the known BH and Born amplitudes. This is summarized by the following expression:

$$
\begin{aligned}
d^{5} \sigma^{\exp }\left(\mathrm{q}, \mathrm{q}^{\prime}, \epsilon, \theta, \varphi\right) & =d^{5} \sigma^{B H+B o r n}\left(\mathrm{q}, \mathrm{q}^{\prime}, \epsilon, \theta, \varphi\right) \\
& +\phi \mathrm{q}^{\prime} \Psi_{0}(\mathrm{q}, \epsilon, \theta, \varphi)+\mathcal{O}\left(\mathrm{q}^{\prime 2}\right)
\end{aligned}
$$

where $d^{5} \sigma$ is a notation for the differential cross section $d^{5} \sigma / d \mathrm{k}_{\text {lab }}^{\prime}\left[\mathrm{d} \Omega_{\mathrm{e}}\right]_{\mathrm{lab}}\left[\mathrm{d} \Omega_{\mathrm{p}}\right]_{\mathrm{CM}}$ wherein $\mathrm{k}_{\text {lab }}^{\prime}$ is the absolute value of the outgoing electron momentum in the laboratory, $\mathrm{q}$ and $\mathrm{q}^{\prime}$ are the absolute values of the threemomenta of the virtual and real photons (in the photonproton center of mass (CM) system) respectively and $\epsilon$ is the virtual photon polarization. $\theta$ is the angle between the real and virtual photon in the $\mathrm{CM}$ system and $\varphi$ is the angle between the electron and the photon-proton plane. $\phi$ stands for a phase space factor. $\Psi_{0}(\mathrm{q}, \epsilon, \theta, \varphi)$ is the leading term in the expansion in powers of the real photon momentum $\mathrm{q}^{\prime}$. It contains the dynamical internal structure information of the proton, expressed by 6 GPs. The latter are denoted by $P^{\left(\rho^{\prime} L^{\prime}, \rho L\right) S}(\mathrm{q})$ where $L\left(L^{\prime}\right)$ are the initial (final) photon orbital angular momentum, $\rho\left(\rho^{\prime}\right)$ the type of multipole transition (0 for Coulomb, 1 for magnetic), and $S$ distinguishes between non-spin-flip $(S=0)$ and spin-flip $(S=1)$ transitions at the nucleon side. One has 2 non-spin-flip GPs, $P^{(01,01) 0}, P^{(11,11) 0}$, proportional to $\alpha_{E}$ and $\beta_{M}$ at $Q^{2}=0$ respectively, and 4 spin-flip GPs, $P^{(11,11) 1}, P^{(11,00) 1}, P^{(11,02) 1}, P^{(01,12) 1}$. The GPs are functions of $\mathrm{q}$ ( or $\tilde{Q}^{2}=\left.Q^{2}\right|_{\mathrm{q}^{\prime}=0}$ ). In an unpolarized measurement, $\Psi_{0}(\mathrm{q}, \epsilon, \theta, \varphi)$ can be written as:

$$
\begin{gathered}
\Psi_{0}(\mathrm{q}, \epsilon, \theta, \varphi)=v_{1}(\theta, \varphi, \epsilon)\left(P_{L L}(\mathrm{q})-\mathrm{P}_{\mathrm{TT}}(\mathrm{q}) / \epsilon\right) \\
+v_{2}(\theta, \varphi, \epsilon) P_{L T}(\mathrm{q})
\end{gathered}
$$

where $v_{1}(\theta, \varphi, \epsilon), v_{2}(\theta, \varphi, \epsilon)$ are known kinematical factors and $P_{L L}(\mathrm{q}), P_{T T}(\mathrm{q})$ and $P_{L T}(\mathrm{q})$ are structure functions defined by:

$$
\begin{aligned}
P_{L L}= & -2 \sqrt{6} m G_{E} P^{(01,01) 0} \\
P_{T T}= & 3 G_{M} \mathrm{q}^{2}\left(\sqrt{2} P^{(01,12) 1}-P^{(11,11) 1} / \tilde{q}_{0}\right) \\
P_{L T}= & \sqrt{\frac{3}{2}} \frac{m \mathrm{q}}{\tilde{Q}} G_{E} P^{(11,11) 0} \\
& +\frac{\sqrt{3}}{2} \frac{\tilde{Q}}{q} G_{M}\left(P^{(11,00) 1}+\frac{\mathrm{q}^{2}}{\sqrt{2}} P^{(11,02) 1}\right)
\end{aligned}
$$

where $m$ stands for the proton mass, $G_{E}$ and $G_{M}$ denote the form factors evaluated at $\tilde{Q}^{2}$ and $\tilde{q}_{0}$ is the CM virtual photon energy at $\mathrm{q}^{\prime}=0$.

Absolute cross sections $d^{5} \sigma^{\text {exp }} 12$ 14 have been measured using the three-spectrometer facility 15] of the A1 collaboration at the $855 \mathrm{MeV}$ Mainz Microtron MAMI. The scattered electron and the recoiling proton were detected in coincidence with two of the high-resolution magnetic spectrometers. The photon production process was selected by a cut on the missing mass around zero, which was possible thanks to the excellent resolution of the facility (momentum resolution of $10^{-4}$ and angular resolution better than $3 \mathrm{mrad}$ ). Liquid hydrogen served as a target, contained in a target cell of $49.5 \mathrm{~mm}$ total length with a havar wall thickness of $9 \mu \mathrm{m}$. The use of typical electron currents of $30 \mu \mathrm{A}$ yielded a luminosity of $\mathcal{L}=4 \cdot 10^{37} \mathrm{~cm}^{-2} \mathrm{~s}^{-1}$.

In this first VCS experiment below pion threshold, the five-fold differential cross section was measured in a wide angular range at 5 values of the photon momentum $\mathrm{q}^{\prime}$ : 33.6, 45.0, 67.5, 90.0, and $111.5 \mathrm{MeV} / \mathrm{c}$. Two kinematical variables were kept fixed, namely the virtual photon momentum, $\mathrm{q}=600 \mathrm{MeV} / \mathrm{c}\left(\tilde{Q}^{2}=0.33 \mathrm{GeV}^{2}\right)$ and the polarization $\epsilon=0.62$. The out-of-plane angle $\varphi$ range is determined by the acceptance of the two spectrometers around $0^{\circ}$ and $180^{\circ}$. To ease the presentation (see Fig. 1 ), the data are plotted with $\theta$ ranging from $-180^{\circ}$ to $+180^{\circ}$, the negative values correspond to $\varphi=180^{\circ}$. The wide range of $\theta$ from $-141^{\circ}$ to $+6^{\circ}$ was covered by only changing the setting of the proton spectrometer. This angular range covers the backward direction relative to the incoming and outgoing electron. Here the VCS contributions are dominant because the $\mathrm{BH}$ radiation is mainly emitted in the electrons directions.

The influence of the GPs gives rise to a small deviation of the measured cross section from the $\mathrm{BH}+\mathrm{Born}$ cross section. Theoretically it is expected to be about $10 \%$ at the highest $\mathrm{q}^{\prime}$ value. The cross sections at each bin are determined within a statistical accuracy of $3 \%$; however, a careful analysis of possible systematic errors on the above deviation is of particular importance. (1) The proton form factors are not exactly known. Consequently, we also measured the absolute elastic scattering cross section for each kinematical setting of the VCS experiment. These measurements validate the use of the form factor parametrization from Höhler 16] at a precision better than $\pm 1 \%$. (2) The luminosity and the detector efficiencies are controlled within the same accuracy. (3) The radiative corrections, which are of the order of $20 \%$ of the cross section, have been calculated by Vanderhaeghen et al. [17 taking into account all the diagrams up to order $\alpha^{4}$ in the VCS cross section. The corresponding systematic uncertainties are estimated to equal $\pm 2 \%$. (4) An extensive Monte Carlo simulation 18] has been performed in order to verify the missing mass spectra and to determine the solid angles within an accuracy of $\pm 2 \%$. The code generates events according to the $\mathrm{BH}+\mathrm{Born}$ cross section and takes into account all resolution deteriorating effects and real photon radiations. (5) While the uncertainties (1)-(4) are constant over the angular range of the real photon, small imperfections in the spectrometer optics calibration produce distortions of the angular distributions: these effects lead to an estimated change in absolute cross section of $\pm 2.5 \%$.

Fig. 1 shows the measured differential cross sections 
(with statistical errors only) as a function of $\theta$ for 5 values of the real photon momentum. The cross sections $d^{5} \sigma^{B H+B o r n}$, calculated using the Höhler form factors, are presented by the solid lines. At the smallest photon momentum, $\mathrm{q}^{\prime}=33.6 \mathrm{MeV} / \mathrm{c}$, the agreement between the data and $d^{5} \sigma^{B H+B o r n}$ is excellent, while with increasing $\mathrm{q}^{\prime}$ one observes growing deviations from this known cross section. This represents the effect of the polarizabilities.

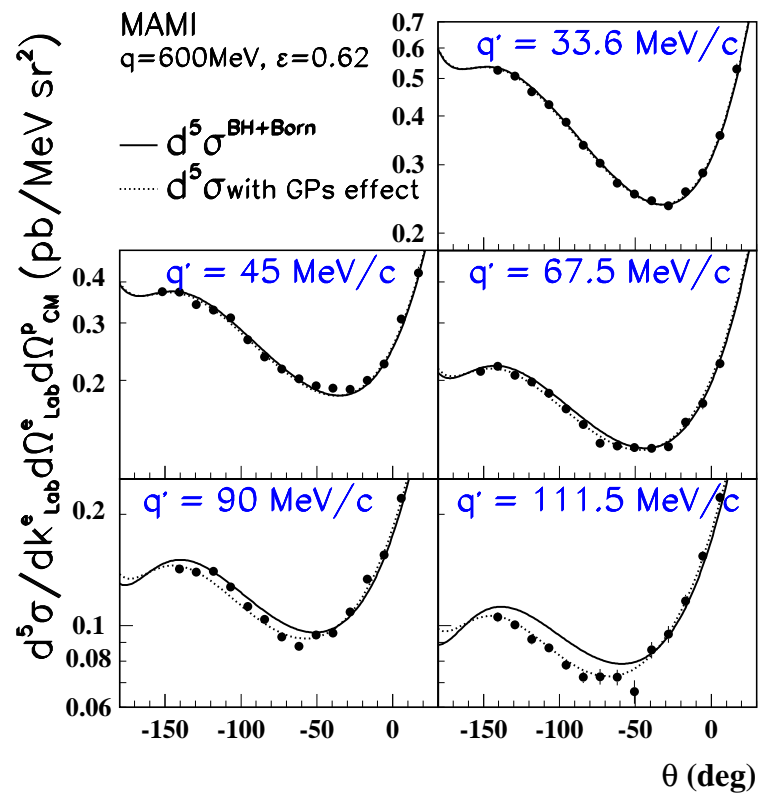

FIG. 1. Differential cross section for the reaction $e p \rightarrow e p \gamma$ measured at MAMI as a function of $\theta$ for $\mathrm{q}=600 \mathrm{MeV} / \mathrm{c}$ or $\tilde{Q}^{2}=0.33 \mathrm{GeV}^{2}, \epsilon=0.62$ and for five values of the real photon momentum $\mathrm{q}^{\prime}$. The known cross section $d^{5} \sigma^{B H+B o r n}$ is indicated by the solid lines. The experimental data points deviate from the solid lines as $\mathrm{q}^{\prime}$ increases; this is the effect of the proton polarizabilities. The dotted lines represent the theoretical cross sections including the effect of the GPS, determined in this experiment.

Fig. 2 shows $\left(d^{5} \sigma-d^{5} \sigma^{B H+B o r n}\right) / \phi \mathrm{q}^{\prime}$ as a function of the real photon momentum $\mathrm{q}^{\prime}$ at the 14 measured angles $\theta$, of which the intercept with the ordinate axis directly yields $\Psi_{0}$ in Eq. (1). Several methods were applied in order to determine the intercept, which will be discussed in a forthcoming paper. The methods that were tried, taking into account a possible $\mathrm{q}^{\prime}$ dependence of $\left(d^{5} \sigma-d^{5} \sigma^{B H+B o r n}\right) / \phi \mathrm{q}^{\prime}$, all show that this dependence is weak. As such, here the most simple approach was used, i.e. no $\mathrm{q}^{\prime}$ dependence. $\Psi_{0}$ is then determined at each angle $\theta$ by the weighted mean value of the data at the 5 photon energies. Fig. 3 presents the resulting values of $\Psi_{0} / v_{2}$ as a function of $v_{1} / v_{2}$ (cf. Eq. (2)). The data are well aligned, this allows us to extract the two structure functions $P_{L L}-P_{T T} / \epsilon$ and $P_{L T}$ as the slope and intercept, respectively, of a linear fit to the data.

Table 1 shows the results with a statistical error and two systematic errors, the first one corresponds to the normalisation of the angular distributions, the second one stems from the distortion of the distributions. All these effects will be evaluated in detail in a forthcoming paper. The other methods for obtaining the combinations give within the error bars compatible results with the ones that are presented here. The dotted lines in Fig. 1 are the theoretical cross sections calculated using the two structure functions measured in this experiment.

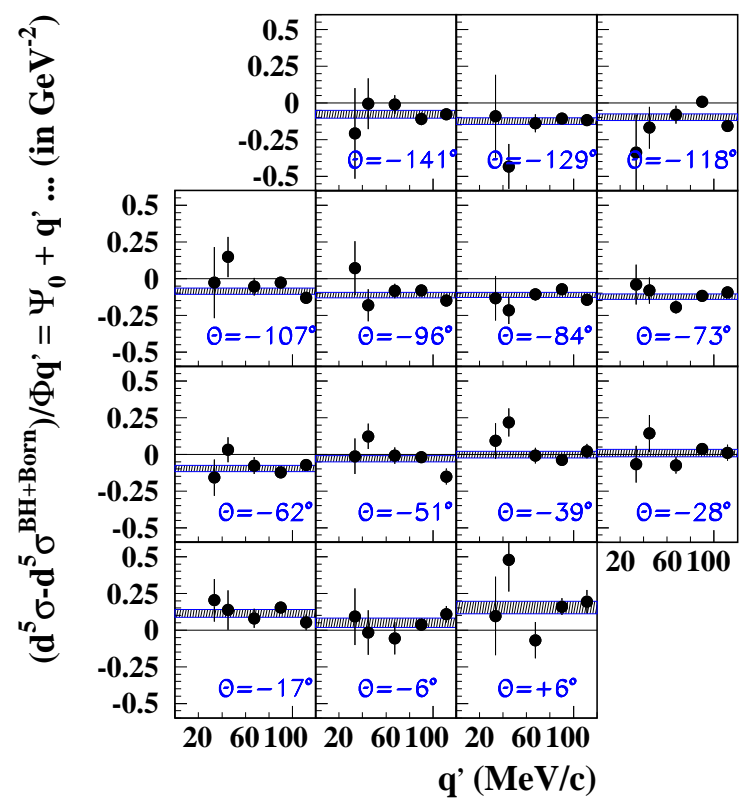

FIG. 2. $\left(d^{5} \sigma-d^{5} \sigma^{B H+B o r n}\right) / \phi \mathrm{q}^{\prime}$ measured as function of the real photon momentum $\mathrm{q}^{\prime}$ at 14 angles $\theta$. The intercept with the ordinate is $\Psi_{0}$. The shaded bands represent the uncertainty in the extrapolation to $\mathrm{q}^{\prime}=0$ by a constant.

Our results for the two structure functions are also compared in Table 1 with different model predictions: a heavy-baryon chiral perturbation theory calculation (HBChPT) [6], the linear sigma model (LSM) [7], an effective lagrangian model (ELM) [8] and two non relativistic constituent quark models (NRCQM) [9,10]). The results seem to favour the HBChPT which at least for $P_{L T}$ may not be so surprising since this approach is based on chiral symmetry and therefore should correctly describe the pion cloud which dominates $P^{(11,00) 1}$ and $P^{(11,02) 1}$, as it was pointed out in the introduction. Note that the LSM model also respects chiral symmetry but it contains otherwise too restrictive hypotheses. The apparent agreement of the NRCQM models for $P_{L T}$ is accidental. It is known that at $Q^{2}=0$ these models overshoot the experimental value of $P^{(11,11) 0}$ by at least a factor 2 .

To summarize, results are reported on the first VCS experiment which allows the determination of two dynamical structure functions which are a linear combination of the GPs. The measurement has been performed at MAMI at $Q^{2}=0.33 \mathrm{GeV}^{2}$ and gives results com- 
patible with chiral perturbation predictions. Two other experiments, aiming at the determination of the same structure functions are performed at different $Q^{2}$ : the Jefferson Lab. experiment 19 at $Q^{2}=1$ and $2 \mathrm{GeV}^{2}$ and the MIT-Bates experiment [20] at $Q^{2}=0.05 \mathrm{GeV}^{2}$. However, to measure independently the 6 GPs and separate non-spin-flip and spin-flip contributions, it will be necessary to perform double polarization experiments.

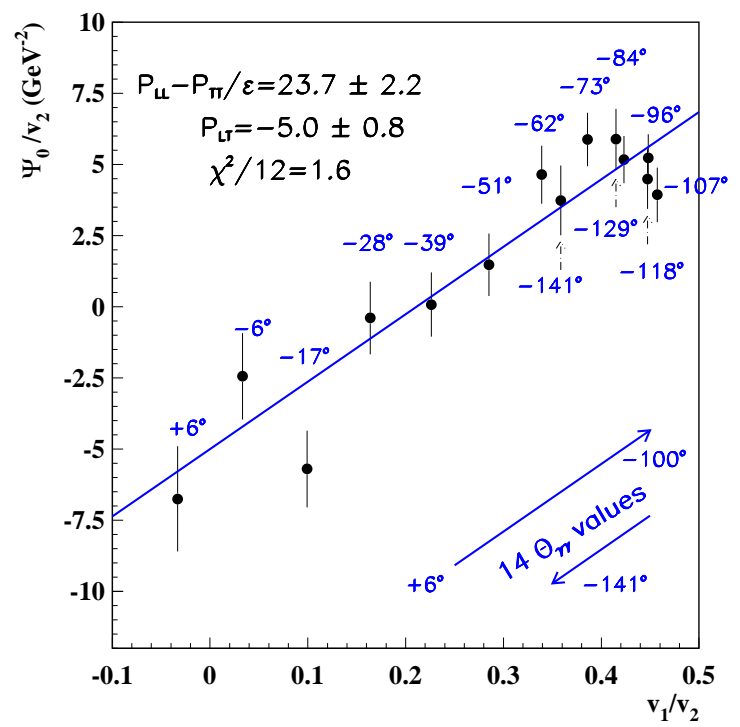

FIG. 3. $\Psi_{0} / v 2$ as a function of $v 1 / v 2$. The errors are statistical only. The line represents the linear fit to the data according Eq. (2) with the values for $P_{L L}-P_{T T} / \epsilon$ and $P_{L T}$ shown in the graph.

The authors wish to acknowledge the excellent support of the accelerator group of MAMI. This work was supported in part by the French CEA and CNRS/IN2P3, the Deutsche Forschungsgemeinschaft (SFB 201), the FWOFlanders (Belgium), the BOF-Gent University, the European Commission ERB FMRX-CT96-0008, the US DOE and the US NSF.
[1] B.E. MacGibbon, G. Garino, M.A. Lucas, A.M. Nathan, G. Feldman, B. Dolbilkin, Phys. Rev. C52, 2097 (1995).

[2] P.A.M. Guichon and M. Vanderhaeghen, Progress in Particle and Nuclear Physics Vol. 41, 125 (1998).

[3] F.E. Low, Phys. Rev. 110, 974 (1958).

[4] P.A.M. Guichon, G.Q. Liu and A.W. Thomas, Nucl. Phys. A591, 606 (1995).

[5] D. Drechsel, G. Knchlein, A. Metz and S. Scherer, Phys. Rev. C55, 424 (1997); D. Drechsel, G. Knchlein, A. Yu Korchin, A. Metz and S. Scherer, Phys. Rev. C57, 941 (1998).

[6] T.R. Hemmert, B.R. Holstein, G. Knöchlein and S. Scherer, Phys. Rev. D55, 2630 (1997); Phys. Rev. Lett. 79, 22 (1997), T.R. Hemmert, B.R. Holstein, G. Knöchlein and D. Drechsel, nucl-th/9910036.

[7] A. Metz and D. Drechsel, Z. Phys. A356, 351 (1996); Z. Phys. A359, 165 (1997).

[8] M. Vanderhaeghen, Phys. Lett. B368, 131996.

[9] G.Q. Liu, A.W. Thomas and P.A.M. Guichon, Aust. J. Phys. 49, 905 (1996).

[10] B. Pasquini, S. Scherer and D. Drechsel, in preparation.

[11] J.F.J. van den Brand et al., Phys. Rev. D52, 4868 (1995).

[12] D. Lhuillier, Ph.D. thesis, Caen, dapnia/sphn-97-01T.

[13] J. Roche, Ph.D. thesis, Clermont, dapnia/sphn-98-06T.

[14] J.M. Friedrich, Ph.D. thesis, Mainz University.

[15] K.I. Blomquist et al., Nucl. Instr. and Meth. A403, 263 (1998).

[16] G. Höhler, E. Pietarinen, I. Sabba-Stefanescu, F. Borkowski, G.G. Simon, V.H. Walther and R.D. Wendling, Nucl. Phys. B114, 505 (1976) and private communication.

[17] M. Vanderhaeghen, J.M. Friedrich, D. Lhuillier, D. Marchand, L. Van Hoorebeke and J. Van de Wiele, Phys. Rev. D62, 014013 (2000).

[18] L. Van Hoorebeke, in preparation.

[19] P.Y. Bertin, P.A.M. Guichon and Ch. Hyde-Wright, spokespersons TJNAF proposal, E-93-050, (1993).

[20] J. Shaw and R. Miskimen, spokespersons MIT-Bates Proposal, (1997).

\footnotetext{
${ }^{1 *}$ corresponding author, electronic address: ndhose@cea.fr

${ }^{2 \dagger}$ present address: College of William and Mary, Williamsburg, Virginia 23187-8795, U.S.A.

${ }^{3 \ddagger}$ present address: Florida International University, Miami, Florida 33199, U.S.A.

${ }^{4 \S}$ present address: IPN d'Orsay, F91406 Orsay Cedex, France

${ }^{5 * *}$ present address: ISN de Grenoble, F38026 Grenoble Cedex, France

${ }^{6 \dagger \dagger}$ present address: Laboratoire SUBATECH, F44307 Nantes cedex 3, France

${ }^{7 \ddagger \ddagger}$ present address: University of Glasgow, Dept. of Physics \& Astronomy, Glasgow G12 8QQ, Scotland, UK
} 
TABLE I. The structure functions determined in this experiment and compared to model predictions at $Q^{2}=0.33$ $\mathrm{GeV}^{2}$ and $\epsilon=0.62$. The first error is statistical only, while the two others are systematic as commented in the text. Previous results[1] at $Q^{2}=0$ and comparison to the same models are also presented.

\begin{tabular}{|c|c|c|}
\hline & $\begin{array}{c}P_{L L}\left(Q^{2}\right)-\frac{1}{\varepsilon} P_{T T}\left(Q^{2}\right) \\
\left(\text { in } \mathrm{GeV}^{-2}\right)\end{array}$ & $\begin{array}{c}P_{L T}\left(Q^{2}\right) \\
\left(\text { in } \mathrm{GeV}^{-2}\right)\end{array}$ \\
\hline \multicolumn{3}{|c|}{$Q^{2}=0.33 \mathrm{GeV}^{2}$} \\
\hline This experiment & 23.7 & -5.0 \\
\hline & $\pm 2.2 \pm 0.6 \pm 4.3$ & $\pm 0.8 \pm 1.1 \pm 1.4$ \\
\hline HBChPT 6 & 26.0 & -5.3 \\
\hline LSM 7] & 11.5 & 0.0 \\
\hline ELM 8 & 5.9 & -1.9 \\
\hline NRCQM [9] & 11.1 & -3.5 \\
\hline NRCQM 10 & 14.9 & -4.5 \\
\hline \multicolumn{3}{|c|}{$Q^{2}=0 \mathrm{GeV}^{2}$} \\
\hline Old experiments $\mathbb{1}$ & 81.0 & -7.0 \\
\hline & $\pm 5.4 \pm 3.3$ & $\mp 2.7 \mp 1.7$ \\
\hline HBChPT 6 & 83.5 & -4.2 \\
\hline $\operatorname{LSM} 7$ & 49.8 & +6.6 \\
\hline ELM & 47.8 & -5.2 \\
\hline NRCQM ]9 & 37.1 & -13.1 \\
\hline NRCQM 10] & 37.0 & -15.8 \\
\hline
\end{tabular}

